\title{
Heat transfer and air temperature measurements of an impinging synthetic air jet
}

\author{
A. McGuinn ${ }^{1}$, T. Persoons ${ }^{1}$, T.S. O’Donovan ${ }^{2}$, D.B. Murray ${ }^{1}$ \\ ${ }^{1}$ Department of Mechanical and Manufacturing Engineering, Trinity College Dublin, \\ Dublin, Ireland, mcguinta@,tcd.ie \\ ${ }^{2}$ School of Engineering \& Physical Sciences, Heriot-Watt University ,Edinburgh, UK, \\ T.S.O'Donovan@,hw.ac.uk
}

\begin{abstract}
Synthetic jets are a relatively new technology that can achieve high rates of heat transfer. They have been shown to out-perform comparable steady impinging jets without the need for an external air supply system. The current experimental research is concerned with the mean and fluctuating heat transfer and local air temperature distribution of an impinging synthetic jet. Tests were conducted for Reynolds numbers from 1000 to 3000, and nozzle to impingement surface spacings of 2 and 6 diameters. The stroke length was maintained constant and equal to 8 diameters in all the reported data. The results obtained show a strong correspondence between the local heat transfer and air temperatures. The periodicity of the synthetic air jet flow characterise the surface heat flux and local fluid temperature behaviour in the stagnation region. At larger radial distances and Reynolds numbers the influence is less significant however.
\end{abstract}

\section{Introduction}

Impinging synthetic air jets can be used to transfer heat in applications ranging from the cooling of manufacturing processes, electronics, turbomachinery. This latter application is increasingly important as current trends in electronic components show a continuous increase in heat flux densities as both processor clock frequency and the number of transistors required for a given implementation grows. This increase has led to an even greater need for more efficient and higher density heat removal in closely packed systems; this need is predicted to continue to grow by a factor of two every four years for the foreseeable future $[2,3]$. While forcing more air through the system or designing geometrically elaborate heat sinks can sometimes meet increasing thermal demands, there are a number of heavy penalties for such implementations. These can include a significant cost increase associated with increasingly elaborate solutions, larger physical thermal design packages when space is at a premium in today's micro-scale electronics applications and a greater level of system noise from fans etc. It is for this reason that we must look to new technologies such as the synthetic jet to reduce both the cost per watt of heat dissipated and the amount of environmental noise produced by these systems.

Synthetic jets operate on a simple principle; a flexible membrane forms one side of a partially enclosed chamber. Opposite to the membrane is an opening, such as an orifice or nozzle. A piezoelectric diaphragm or a mechanical actuator can be used to oscillate the membrane and periodically force air into and out of the opening. The result of this is the formation of a non-zero mean streamwise pulsating jet in front of the orifice which can be directed at a heated surface to significantly enhance heat transfer. 
The main variables for synthetic jet impingement heat transfer are the excitation frequency, stroke length and the nozzle height above the impingement surface. Comprehensive reviews of heat transfer to impinging synthetic air jets have been conducted by Gillespie et al. [4], Wang et al. [5] and Pavlova and Amitay [6] amongst others. Campbell et al. [7] conducted a review of heat transfer to an impinging synthetic jet for a wider range of parameters that included varying the number of jets in a jet array, various levels of confinement and the addition of cross flow. Gallas et al. [8] presented an extensive review of numerical investigations that have been conducted in the area of lumped element modelling of piezoelectric-driven synthetic jet actuators. Campbell et al. [9] also conducted experiments to determine the effectiveness of synthetic air microjets in cooling packaged thermal test chips and a laptop computer processor. Kercher et al. [1] investigated the applicability of miniaturized synthetic jet technology to the area of thermal management of microelectronic devices and directly compared the cooling performance of these microjets with standard cooling fans. Gillespie et al. [4] investigated the effects of a small-scale, rectangular synthetic jet on the local convective heat transfer from a heated surface and also measured the velocity field between the jet orifice and the target plate[10, 11].

Many investigations have been undertaken into the optimum operating parameters of the synthetic jet; one by Gallas et al. [12] optimised the jet with respect to variables such as driving frequency, cavity volume, nozzle length and nozzle diameter. It has been documented by Li and Zhong [13] and Kercher et al. [1] that the shape of the heat transfer distribution changes significantly with nozzle to impingement surface spacing. Smith and Swift [14] concluded that there exists a minimum dimensionless stroke length $\mathrm{L}_{0} / \mathrm{D}$ below which no synthetic jet is formed. It is also stated that the far field behaviour of synthetic jets appears to be a function of both $\mathrm{L}_{0} / \mathrm{D}$ and Re; these findings were confirmed in a paper by Holdman and Utturkar [15]. It is for this reason that it is necessary to gain a better understanding of the different heat transfer mechanisms produced by varying stroke length and Reynolds number and their effects on heat transfer.

Comprehensive studies have been undertaken into the fluid flow and heat transfer characteristics of impinging synthetic jets $[1,4,6,13]$. These studies present data highlighting the effect Reynolds number and stroke length has on heat transfer to the synthetic jet by measuring parameters such as flow velocity, turbulence intensity and vorticity. However, little or no research has been undertaken to assess extent to which air temperature influences heat transfer from the impingement surface. Other areas of engineering such as aerospace and power generation rely heavily on temperature difference between the working fluid and heated surface to enhance heat transfer. Significant research has been undertaken which shows how effective this temperature differential can be at transferring heat to a working fluid [16, 17]. Rydholm [16] observed that a greater heat transfer magnitude present further downstream of the jet due to reduced turbulence, this lower turbulence maintained coherence in the cooler core air to a greater distance from the orifice. Jovanovi [17] observed a similar phenomenon where increased turbulence resulting from hole imperfections resulted in lower local heat transfer, however higher overall heat transfer was observed due to the mixing of the cooler jet with the ambient fluid. These papers stop short of taking local fluctuating temperature measurements however it is likely that they would contribute greatly to the understanding of the types of flow and mechanisms involved in heat transfer.

Although many studies have investigated the heat transfer to an impinging synthetic jet as a function of Reynolds number, the vast majority of these tests have been conducted using a 
variety of jet stroke length. It is of great importance that a better understanding of the effect of varying Reynolds number on heat transfer independent of stroke length be obtained and due to the potential of the synthetic jet to be used in confined environments such as inside computers. It is increasingly important that these parameters can be optimised for low jet to surface spacings which commonly occur within these types of small electronics packages. Furthermore, little or no data is available for combined fluctuating heat transfer and fluctuating air temperature profiles. The present study sets out to provide detailed local heat transfer profiles, both time-averaged and fluctuating. time-resolved simultaneous surface heat transfer rate and local fluid temperature measurements will be analysed and presented for a synthetic jet impinging onto a heated surface at close range.

\section{Experimental Setup}

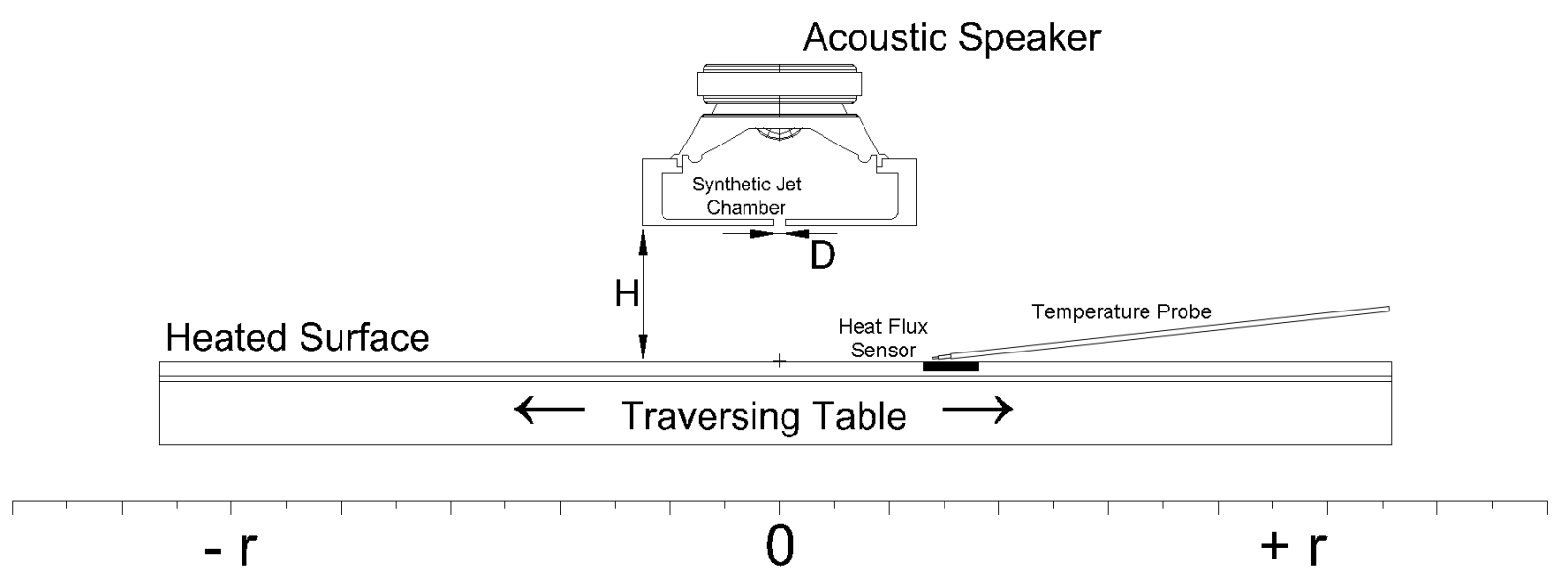

Figure 1: Experimental Rig

The two main components of the experimental rig are the synthetic jet actuator and the heated impingement surface. As indicated in figure 1, the impingement surface is instrumented with two sensors to measure the heat flux locally with high spatial resolution. The heated surface is mounted on a computer-controlled traversing table which allows the sensors to be displaced relative to the synthetic air jet flow. A high response temperature probe is positioned $0.3 \mathrm{~mm}$ $(+/-0.1 \mathrm{~mm})$ above the hot film sensor to measure the local fluid temperature, as indicted above.

The operation of the synthetic jet relies primarily on an acoustic speaker mounted on an enclosed cavity with an orifice plate on the opposing side to provide the entrainment path for the working fluid. A driving frequency for the speaker is provided by a TTi TG315 Signal Generator, and the signal is amplified using a Kemo ${ }^{\circledR} 40$ Watt power amplifier. The speaker is supplied with a sinusoidal wave of specific amplitude and frequency so as to obtain the desired stroke length and Reynolds number.

The jet impinges on a surface that consists of a $5 \mathrm{~mm}$ thick flat copper plate measuring $425 \mathrm{~mm} \times 550 \mathrm{~mm}$. To the underside of the plate a silicon rubber heater mat is glued with a thin layer of adhesive. The underside of the plate is insulated from the surroundings. The plate assembly is such that it approximates a uniform wall temperature boundary condition. The system is typically operated and maintained at a surface temperature of $40^{\circ} \mathrm{C}$.

An RdF Micro-Foil ${ }^{\circledR}$ heat flux sensor uses a differential thermopile to measure the time-averaged surface heat transfer; the temperature differential across a known thermal 
barrier is used to calculate the heat flux as indicated:

$$
\ddot{q}=k_{s} \frac{\Delta T}{\delta}
$$

where $k_{s}$ is the thermal conductivity of the thermal barrier (kapton) and $\Delta T$ is the temperature differential across the thickness $(\delta)$ of the barrier. A single pole T-type thermocouple is also embedded in this sensor to measure the local surface temperature.

A Senflex ${ }^{\circledR}$ hot film sensor operates in conjunction with a Constant Temperature Anemometer (CTA) to measure the local fluctuating heat flux from the plate to the impinging jet. The hot film is maintained at a slight overheat $\left(\approx 1^{\circ} \mathrm{C}\right)$ above that of the copper plate using a Dantec StreamLine CTA. The power required to maintain the film at this overheat is equal to the heat actively being dissipated from the film. The CTA essentially acts as a Wheatstone bridge where the hot film acts as one resistor in the bridge. The resistance of the film varies with temperature and therefore, the film temperature can be controlled by varying a decade resistance which forms another arm of the Wheatstone bridge. The square of the voltage required to maintain the film at a constant temperature is proportional to the heat transferred to the air as described in equation 2 .

$$
q_{\text {dissipated }} \propto \frac{V_{\text {out }}^{2}}{R}
$$

A Dantec Dynamics Type 55P11 probe is used to measure the fluctuating air temperature at the plate surface immediately above the hot film Sensor. The probe operates in conjunction with a Dantec Dynamics Constant Current Anemometer (CCA) StreamLine Temperature Module. The probe itself consists of a fine platinum plated tungsten wire sensor, $5 \mu \mathrm{m}$ in diameter and $1.5 \mathrm{~mm}$ in length, welded between two prongs. Using theory outlined by Bruun [18] the time constant for such a wire operated in constant current mode at a very low overheat temperature and air velocities as low as $1 \mathrm{~m} / \mathrm{s}$ would be $1 \mathrm{~ms}$. This can be shown using the following equation:

where $\mathrm{M}$ is a time constant.

$$
M=\frac{\rho_{w} c_{w} D^{2}}{4 k_{a} N u}
$$

The heat transfer rig used in this paper is similar to that used by O'Donovan [19]. The mean and fluctuating Nusselt numbers have calculated uncertainties of $5.7 \%$ and $30 \%$ respectively. These uncertainties are based on a worst case scenario where the uncertainty is a percentage of the smallest measurements. A complete calibration and uncertainty analysis for this experimental set-up is presented by O'Donovan [20].

The Synthetic jet was operated at Reynolds numbers of 1000, 2000 and 3000 with a constant dimensionless stroke length of $L / D=8$. Jet operating frequencies were 33,77 and $119 \mathrm{~Hz}$ respectively and tests were conducted at dimensionless spacings of $H / D=2,4$ and 6 . The orifice was round in shape with a diameter of $5 \mathrm{~mm}$ and length of $10 \mathrm{~mm}$.

\section{Results and Discussion}

Data are presented in figures 2 and 3 of the mean and root-mean-square Nusselt number, and the local and root-mean-square fluid temperature distributions for a synthetic air jet impinging at $H / D$ values of 2 and 6 respectively.

As indicated in figure 2(a) the maximum surface heat transfer occurs at the stagnation point and decays with increasing radial distance, greater Reynolds numbers also produce higher levels of heat transfer. This is similar to steady jet impingement, however as reported 


\section{A. McGuinn et al.}

by O'Donovan et al. [18] secondary peak tend to occur in the distribution at a radial location between $\mathrm{r} / \mathrm{D}=1$ and 2 . This does not occur for the synthetic air jet at the lower value of $H / D$ tested but is observed at the higher value of $H / D=6$ as indicated in figure 3(a). For similar stroke length, $\operatorname{Re}=1020$ and $H / D=2$, Valiorgue et al. [10] show a small secondary peak around $r / D=3$, although this study applies a constant wall flux boundary condition. By comparison with particle image velocimetry (PIV) measurements, the peak location coincides with the point where the vortex loses coherence and dissipates into turbulence.

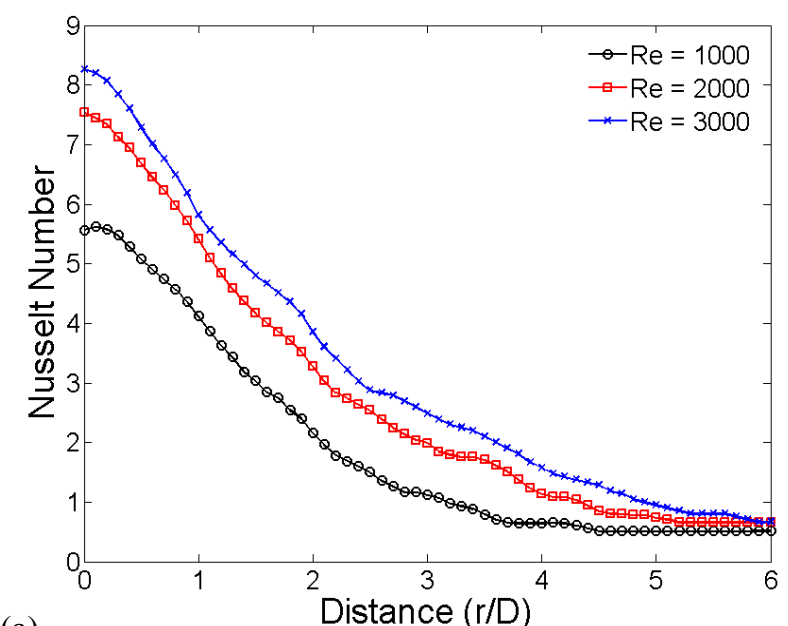

(a)

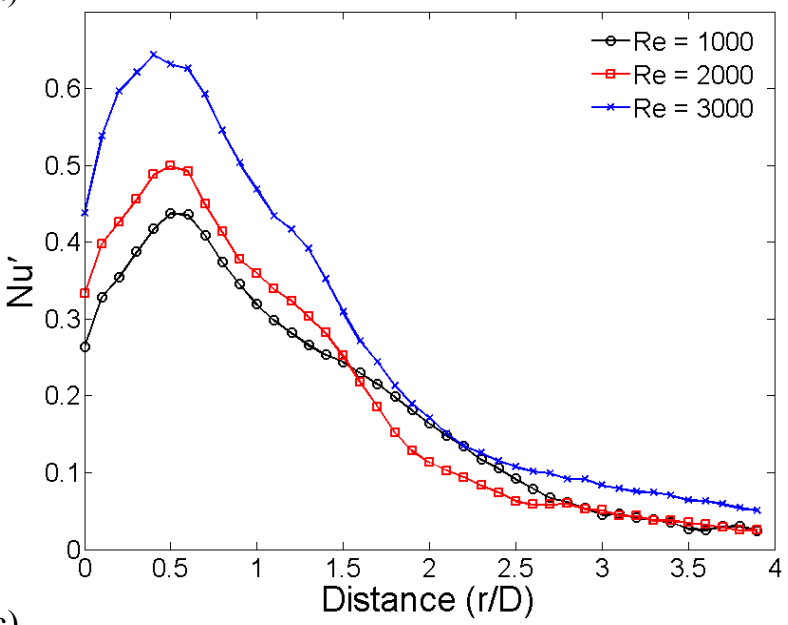

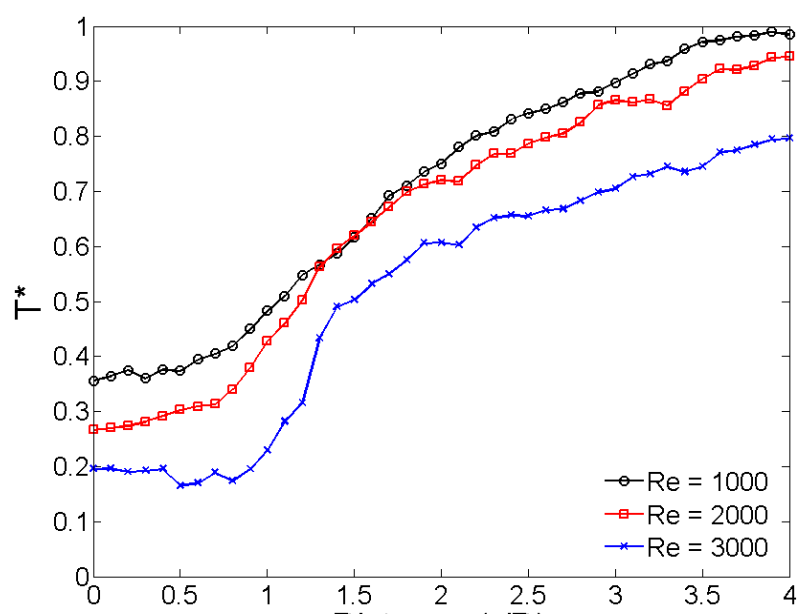

(b)

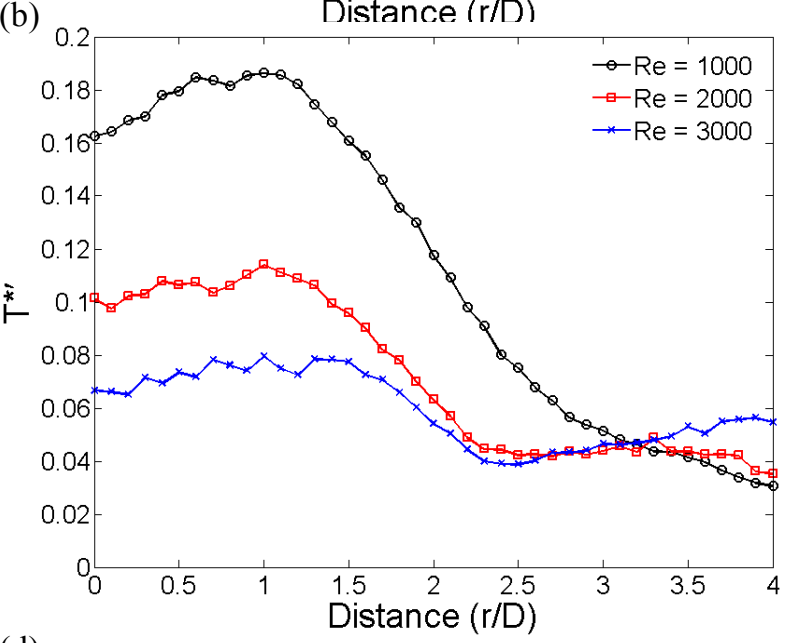

(d)

Figure 2: Nusselt number and local fluid temperature distributions for $\mathrm{H} / \mathrm{D}=2$

It is thought that the ratio between the stroke length and the nozzle to impingement surface spacing influences the magnitude of the secondary peak. A previous study by the authors [10], Valiorgue] has shown that the ratio of stroke length to nozzle-to-surface spacing $L_{0} / H$ determines different flow regimes and heat transfer mechanisms in impinging synthetic jets for low jet-to-surface spacing. A critical ratio of $L_{0} / H=2.5$ has been identified for $H / D=$ 2 , although this critical value is thought to be slightly dependent on $H / D$.

The present study has been conducted for a constant stroke length of $8 D$, and figure 2 presents data for $H / D=2$, consequently these parameters result in a stroke length which is substantially larger than nozzle to surface spacing. In this case the flow does not form vortices which can convect downstream before impingement; instead the main slug flow impinges on the heated surface periodically. This is confirmed by PIV measurements performed by Valiorgue et al. 2009 [10].It has been established by O'Donovan and Murray [21] that the 
magnitude of the secondary peak is proportional to the size and coherence of vortices in the main jet flow. However in this case as the jet has not had space to develop between exit and impingement surface, the vortices do not break up in the wall jet effecting an increase in the heat transfer locally to form secondary peaks in the distribution.

In figure 3 the height of the nozzle above the impingement surface approaches the stroke length; thus allowing the synthetic jet flow to develop vortices before impingement. These vortices then break up in the wall jet which result in an increase in the turbulence and enhance heat transfer. This effect is clear for a Reynolds number of 1000 and less so for the larger values. Why?
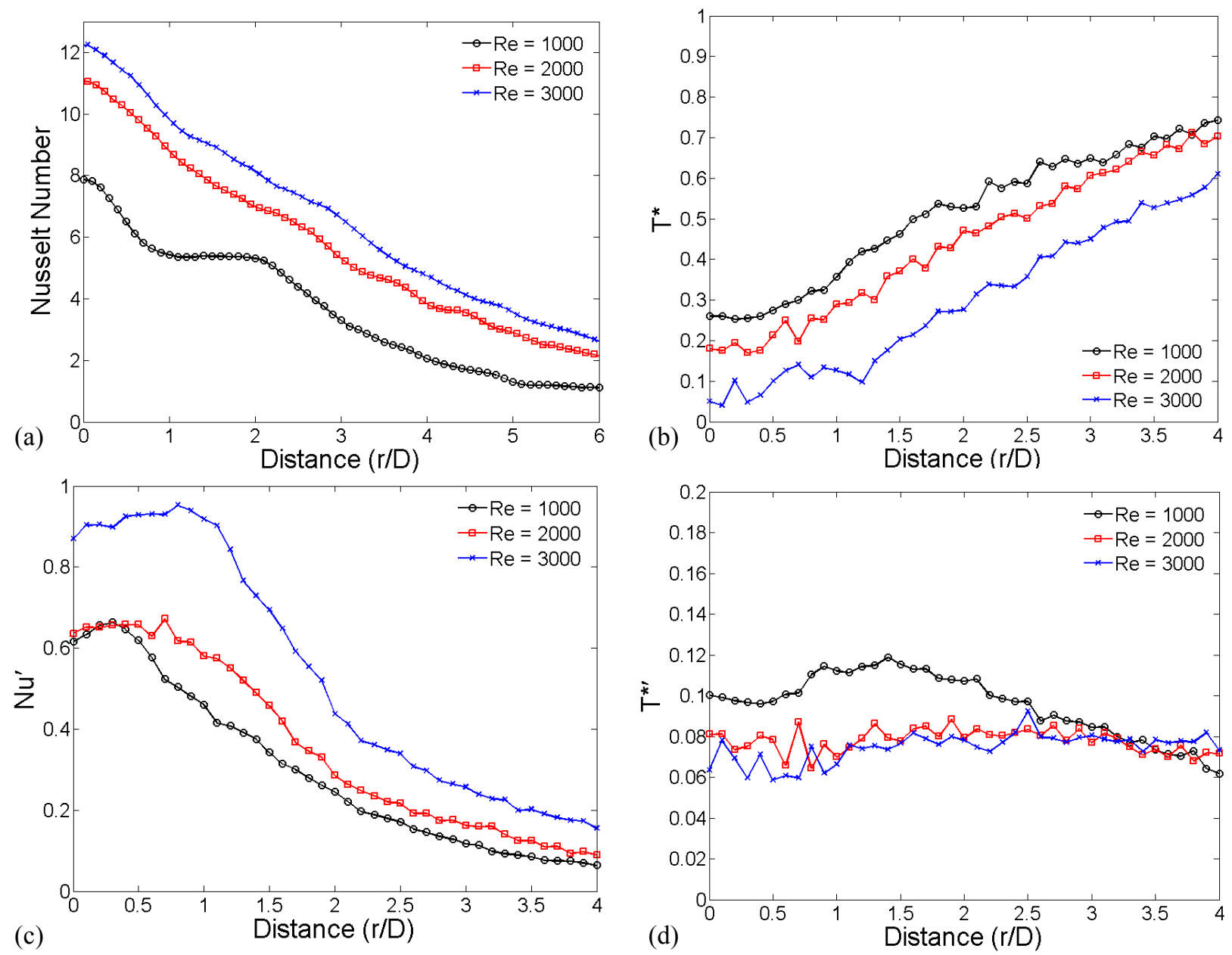

Figure 3: Nusselt number and Local Fluid Temperature Distributions for H/D $=6$

Figures 2(b-d) are used to further understand the heat transfer distributions presented in figure 2(a). The time-averaged and normalised local air temperature distributions (figure 2(b), 3(b)) indicate that the air temperature is low in the stagnation region and increases with increasing radial distance. The higher heat transfer correlates well with the corresponding lower local air temperature; most notably for $H / D=2$ where the local air temperature approaches the temperature of the surface at a radial location of approximately $3.5 \mathrm{D}$. At the higher value of $H / D=6$ however, the local air temperature reaches a maximum of $70 \%$ of the temperature difference within the same radial distance. This is clearly a result of the confinement and increased flow re-circulation at the lower $H / D$ value. This results in lower area-averaged heat transfer due to the increased recirculation of hot air into the cavity, which decreases its cooling effectiveness. At a Reynolds number of 3000 and $H / D=2$ the local air 
temperature is low and constant over a distance of $r / D<1$; beyond this distance the local air temperature increased sharply. This region of low air temperature is attributable to the jet slug flow which maintains a constant low air temperature. Beyond this distance however the ambient air re-circulates and therefore a significant increase in air temperature is experienced. This is consistent with PIV flow measurements presented by McGuinn et al. [22] and Valiorgue et al. [10]. This does not occur at larger values of $H / D$ as the slug flow no longer impinges directly on the heated surface.

Distribution of the root-mean-square Nusselt number are presented in figure 2(c) and 3(c) for $H / D=2$ and 6 respectively. In general the magnitude of the heat transfer fluctuations decrease with increasing radial distance however, at the lower $H / D=2 \mathrm{Nu}$ attains a local minimum at the stagnation point; this is less evident at higher values of $H / D$. This is again indicative of the slug flow impingement which suppresses turbulence in the stagnation region. Overall the magnitudes of the heat transfer fluctuations are much lower at $H / D=2$; this could also indicate that higher levels of confinement and recirculation may suppress the turbulence in an impinging synthetic air jet flow.

The magnitude of the local air temperature fluctuations are presented in figures $2(d)$ and $3(d)$. It can be seen that the magnitude of the fluctuations are high in the stagnation region for $H / D=2$ and decrease with increasing radial distance. In contrast the magnitude of the temperature fluctuations are low and reasonably constant across the profile for $H / D=6$. These temperature fluctuations at $R e=1000$ and $H / D=2$ in particular are much higher in the stagnation region. Since the net mass flux created by the jet at low Reynolds number still effects heat transfer comparable to the higher Reynolds numbers then this results in a substantial increase in the air temperature fluctuations locally. The relative mass flux produced by the impinging synthetic air jet at low $H / D$ is less than that for a jet impinging at a higher $H / D$ or a free jet, this is because the increased confinement produced by the low jet exit to impingement surface spacings are subject to more recirculation of the same air flow. Therefore it is understandable that the temperature fluctuations are elevated at low values of $H / D$. This is further explained by examining time-traces of the local surface heat flux and air temperature, presented in figure 4 and 5. Figure 2(b) and 3(b) show that average fluid temperature is greater for lower Reynolds numbers; this produces a larger temperature differential between ambient temperature and fluid temperature. Consequently ambient air entrained in the jet flow results in the larger fluctuation in the air temperature as can be seen in figure 2(d) and 3(d).

Both the surface heat flux and local air temperature were measured simultaneously and at high temporal resolution (data acquisition frequency of $16,384 \mathrm{~Hz}$ ). Simultaneous time-traces of the local air temperature at four radial locations $(r / D=0,2,3,3.9)$ are compared to negative surface heat flux signals in figures 4 and 5 for a Reynolds numbers of 1000 and 3000 respectively at a $H / D=2$. For the range of signals studied the local air temperature is relatively coherent with the surface heat flux signal. The periodicity of the signals is clear at the stagnation point and in the near wall jet region, however at greater radial distances the signals are less structured indicating a breakdown of the periodic nature of the flow into a more random turbulent flow. At lower Reynolds numbers the signals are more closely aligned indicating that the local air temperature has more of an influence on the resulting surface heat transfer magnitude. The magnitude of the Reynolds number is directly proportional to the frequency for a constant stroke length as used in this study. It is therefore understandable that the coherence between the surface heat flux signal and the air temperature would decrease for higher jet frequencies and velocities as the local velocity of the flow would be more of a factor in affecting the surface heat transfer. As the local air velocity is much lower at greater r/D and 
for the lower Reynolds number jet, it is expected that the local air temperature would respond to the heat transfer signal more completely resulting in higher coherence between the two signals.

Finally there is a slight phase lag between the two signals which is indicative of the convection velocity between the two measurement points. This convection velocity will be in the direction normal to the impingement surface and therefore not in line with the local air flow direction. Further analysis of the signal spectra, coherence and phase are intended to gain further understanding of the effects of confinement, slug length, Reynolds number etc. on the local and area-averaged heat transfer.
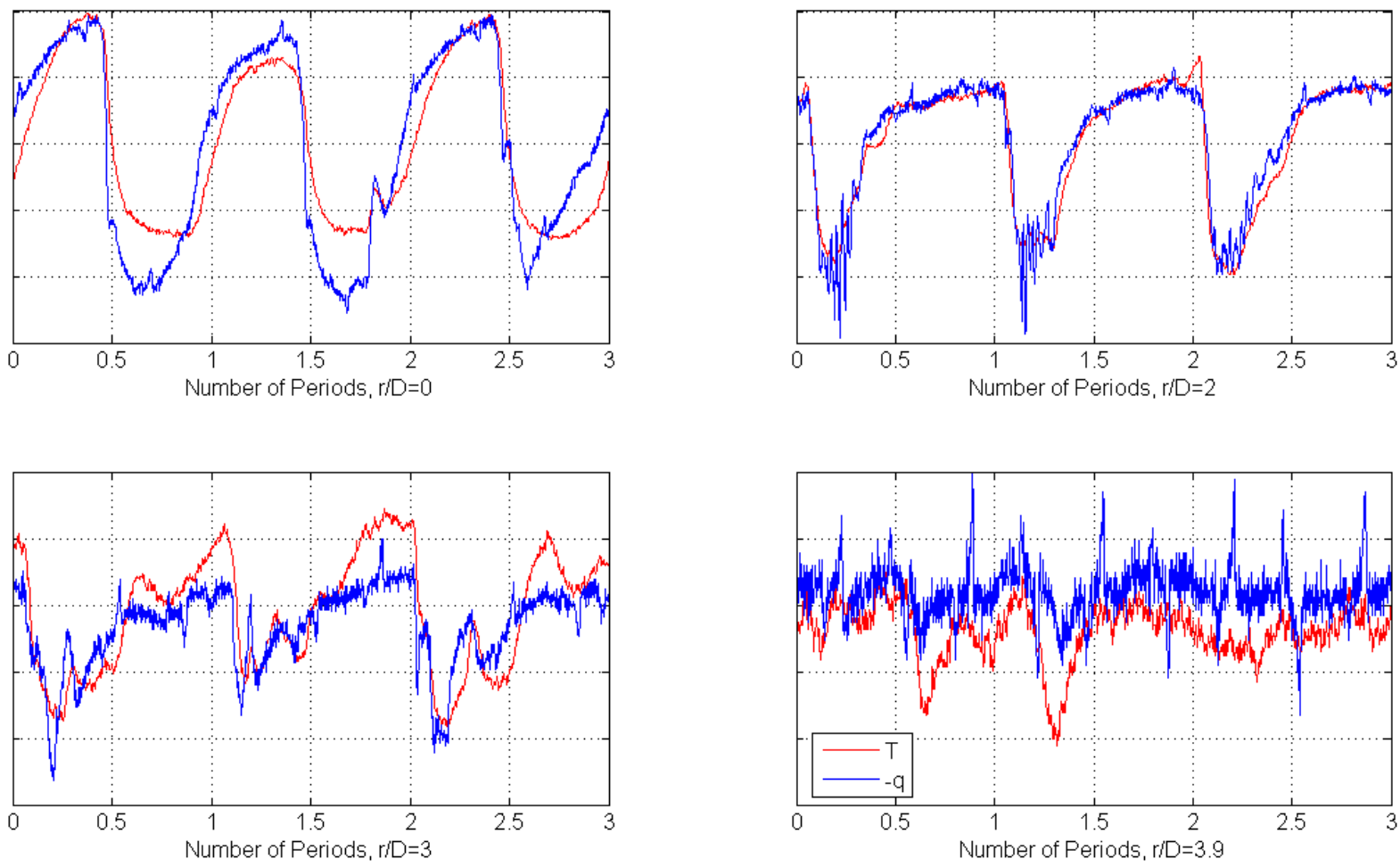

Figure 4: Temperature and negative surface heat flux time-traces for $R e=1000, H / D=2$ (all plots have the same vertical arbitrary scale) 

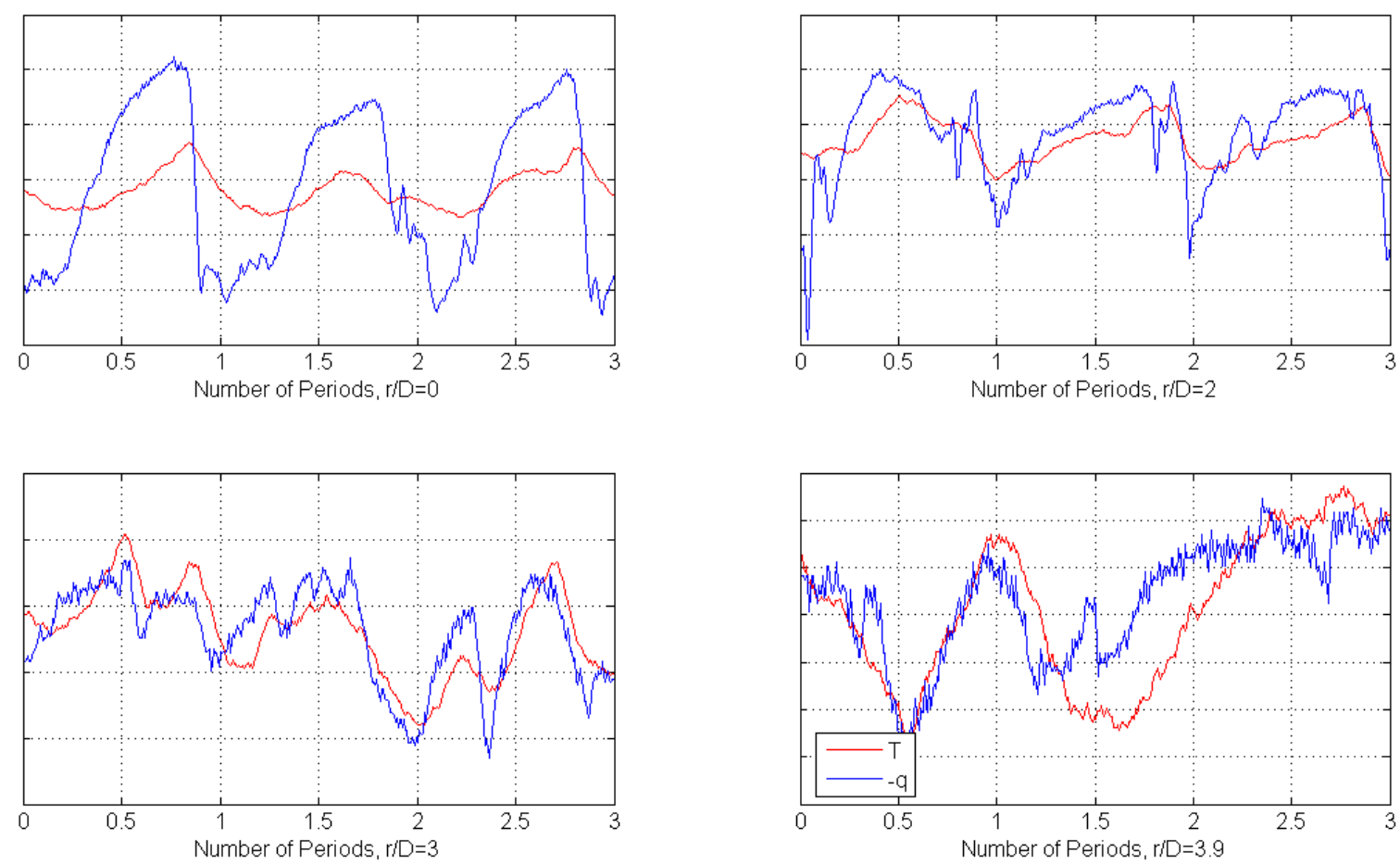

Figure 5: Temperature and negative surface heat flux time-traces for $R e=3000, H / D=2$

\section{Conclusions}

A methodology has been established which has enabled us to measure simultaneous local air temperature and surface heat transfer fluctuation at a both a high special and temporal resolution, using constant temperature anemometry and constant current anemometry. These techniques have provided us with data for an axisymmetric synthetic air jet of various Reynolds number with constant stroke length of $8 D$ impinging normal to a heated surface. It was found that higher heat transfer correlates well with lower local air temperature further out confinement and increased flow re-circulation at lower $H / D$ values result in local air temperature approaching the temperature of the surface with increasing radial distance.

For the given stroke length and low $H / D$, a slug flow impinges and maintains a constant low air temperature within the core, however beyond this increase in air temperature is experienced due to recirculation, confirmed by previous studies [22].

Air temperature fluctuations have been found to be consistently greater at lower Reynolds numbers and lower $H / D$. This is because the lower net mass flux at lower Reynolds numbers still affects heat transfer compared to the higher Reynolds numbers resulting in a substantially increased local air temperature.

Normalised temperature profiles revealed the fluid temperature is greater for lower Reynolds numbers and lower $H / D$ resulting in the larger fluctuations observed in time traces for air temperature and surface heat flux. Time domain analysis shows that at greater radial distance air temperature and surface heat flux signals are more closely aligned indicating that the local air temperature has more of an influence on heat transfer magnitude.

Finally the coherence between the surface heat flux and the local air temperature has been shown to decrease with increasing Reynolds number even though the mean heat transfer increases. It is therefore hypothesised that at higher Reynolds numbers, near-wall velocity has 
more of an influence than local air temperature.

\section{Nomenclature}

$c_{w} \quad$ heat capacity of wire

$D \quad$ jet diameter, $m$

$\delta \quad$ sensor thickness, $m$

$f \quad$ frequency, $\mathrm{Hz}$

$H \quad$ height of nozzle above plate, $m$

$k_{a} \quad$ thermal conductivity of air, $W / m K$

$k_{s} \quad$ thermal conductivity of sensor barrier, $W / m K$

$L_{0} \quad$ jet's stroke length, $m$

$M \quad$ time constant

$\mathrm{Nu} \quad$ Nusselt number, $h D / k$

$\mathrm{Nu}$ ' root-mean-square Nusselt number

$q \quad$ rate of heat transfer, $W$

$\ddot{q} \quad$ heat flux, $W / m^{2}$

$R \quad$ sensor resistance, $\Omega$

$r \quad$ radial distance along plate from geometric centre, $m$

Re Reynolds number, $U D / v$

$\rho \quad$ density, $\mathrm{kg} / \mathrm{m}^{3}$

$\rho_{w} \quad$ density of wire, $\mathrm{kg} / \mathrm{m}^{3}$

$T \quad$ temperature, ${ }^{\circ} \mathrm{C}$

$T^{*} \quad$ normalised air temperature, $\left(T_{\text {air }}-T_{\text {ambient }}\right) /\left(T_{\text {surface }}-T_{\text {ambient }}\right)$

$T^{*}$, normalised fluctuating air temperature, $T_{\text {air }}^{\prime} /\left(T_{\text {surface }}-T_{\text {ambient }}\right)$

$\Delta T$ temperature difference, ${ }^{\circ} \mathrm{C}$

$\mu \quad$ viscosity, $\mathrm{kg} / \mathrm{ms}$

$V_{\text {out }} \quad$ output voltage, $V$

$v \quad$ kinematic viscosity, $\mathrm{m}^{2} / \mathrm{s}$

\section{Acknowledgments}

The authors gratefully acknowledge the technical support staff of the Mechanical and Manufacturing Engineering Department of Trinity College. The project is part funded by SFI in collaboration with the Centre for Telecommunications Value-Chain Research (CTVR).

\section{References}

[1] D. S. Kercher, J.-B. Lee, O. Brand, M. G. Allen, and A. Glezer, "Microjet cooling devices for thermal management of electronics," IEEE Transactions on Components and Packaging Technologies, vol. 26, pp. 359-366, 2003.

[2] A. Allan, D. Edenfeld, W. H. Joyner, Jr., A. B. Kahng, M. Rodgers, and Y. Zorian, "2001 technology roadmap for semiconductors," Computer, vol. 35, pp. 42-53, 2002.

[3] S. H. Gunther, F. Binns, D. M. Carmean, and J. C. Hall, "Managing the impact of increasing microprocessor power consumption," Intel Technology Journal, 2001. 
[4] M. B. Gillespie, W. Z. Black, C. Rinehart, and A. Glezer, "Local convective heat transfer from a constant heat flux flat plate cooled by synthetic air jets," Journal of Heat Transfer, vol. 128, pp. 990-1000, 2006.

[5] Y. Wang, G. Yuan, Y.-K. Yoon, M. G. Allen, and S. A. Bidstrup, "Large eddy simulation (LES) for synthetic jet thermal management," International Journal of Heat and Mass Transfer, vol. 49, pp. 2173-2179, 2006.

[6] A. Pavlova and M. Amitay, "Electronic cooling using synthetic jet impingement," Transactions of the ASME. Journal of Heat Transfer, vol. 128, pp. 897-907, 2006.

[7] S. J. Campbell, Jr., W. Z. Black, A. Glezer, and J. G. Hartley, "Thermal management of a laptop computer with synthetic air microjets," New York, NY, USA, 1998, pp. 43-50.

[8] Q. Gallas, Wang, G., Papila, M., Sheplak, M., Cattafesta, L., "Optimization of Synthetic Jet Actuators," in 41st Aerospace Sciences Meeting \& Exhibition. vol. AIAA 2003-0635 Reno, NV, USA.: American Institute of Aeronautics and Astronautics, 2003.

[9] J. S. Campbell, W. Z. Black, A. Glezer, and J. G. Hartley, "Thermal management of a laptop computer with synthetic air microjets," IEEE InterSociety Conference on Thermal Phenomena, pp. 43 - 50, 1998.

[10] P. Valiorgue, T. Persoons, A. McGuinn, and D. B. Murray, "Heat transfer mechanisms in an impinging synthetic jet for a small jet-to-surface spacing," Experimental Thermal and Fluid Science, vol. 33, pp. 597-603, 2009.

[11] T. Persoons, O’Donovan, T. S., Murray, D. B.," "Heat transfer in adjacent interacting impinging synthetic jets," in ASME Summer Heat Transfer Conference San Francisco (CA), USA, 2009.

[12] Q. Gallas, R. Holman, T. Nishida, B. Carroll, M. Sheplak, and L. Cattafesta, "Lumped element modeling of piezoelectric-driven synthetic jet actuators," AIAA Journal, vol. 41, pp. 240-7, 2003.

[13] M. Li, Zhong, S., "Heat transfer and flow characteristics of impinging synthetic jets," in 9th UK National Heat Transfer Conference Manchester, UK 2005, p. 12.

[14] B. L. Smith and G. W. Swift, "A comparison between synthetic jets and continuous jets," Experiments in Fluids, vol. 34, pp. 467 - 472, 2003.

[15] Y. Utturkar, Holman R., Mittal R., Carroll B., Sheplak M., Cattafesta L., "A Jet Formation Criterion for Synthetic Jet Actuators," in 41st Aerospace Sciences Meeting $\&$ Exhibit Reno, NV, USA: American Institute of Aeronautics and Astronautics, 2003, p. 9.

[16] H. A. Rydholm, "Experimental investigation of the velocity and temperature fields of cold jets injected into a hot crossflow," Burmingham, UK, 1996, pp. 8pp-8pp.

[17] M. B. Jovanovi, H. C. de Lange, and A. A. van Steenhoven, "Effect of hole imperfection on adiabatic film cooling effectiveness," International Journal of Heat and Fluid Flow, vol. 29, pp. 377-86, 2008.

[18] H. H. Bruun, Hot-wire anemometry: principles and signal analysis. Oxford University Press, Walton Street, Oxford OX2 6DP: Oxford University Press, 1995.

[19] T. S. O'Donovan, "Fluid flow and heat transfer of an impinging air jet," in Mechanical \& Manufacturing Engineering. vol. Ph. D.: Trinity College Dublin, 2005.

[20] T. S. O'Donovan, "Fluid flow and heat transfer of an impinging air jet," in Department of Mechanical and Manufacturing Engineering. vol. PhD Dublin: Trinity College Dublin, 2005, p. 145.

[21] T. S. O'Donovan and D. B. Murray, "Jet impingement heat transfer - Part II: A 
temporal investigation of heat transfer and local fluid velocities," International Journal of Heat and Mass Transfer, vol. 50, pp. 3302-3314, 2007.

[22] A. McGuinn, T. Persoons, P. Valiorgue, T. S. O'Donovan, and D. B. Murray, "Heat transfer measurements of an impinging synthetic air jet with constant stroke length," in 5th European Thermal-Sciences Conference, Eindhoven, 2008, p. JET 4. 